\title{
Gastric pneumatosis and portal venous gas: benign findings in hypertrophic pyloric stenosis
}

\author{
Puneet Bhargava $\cdot$ Marguerite Parisi
}

Received: 17 September 2008 /Revised: 12 October 2008 / Accepted: 21 November 2008 / Published online: 9 January 2009

(C) Springer-Verlag 2009

A 6-week-old boy with progressively increasing non-bilious emesis was referred for evaluation of suspected pyloric stenosis. US revealed thickening and elongation of the pylorus, classic for pyloric stenosis, with reverberation artefact from the gastric wall. Echogenic foci compatible with portal venous gas were noted in appropriate distribution within the imaged liver (Fig. 1). Abdominal plain films confirmed gastric pneumatosis as the cause of portal venous gas (Fig. 2).

Gas in the bowel wall in infants is an alarming finding, raising concerns for gut ischemia and necrotizing enterocolitis. Gastric pneumatosis in the absence of ischemia has been reported in gastric outlet obstruction, including that from

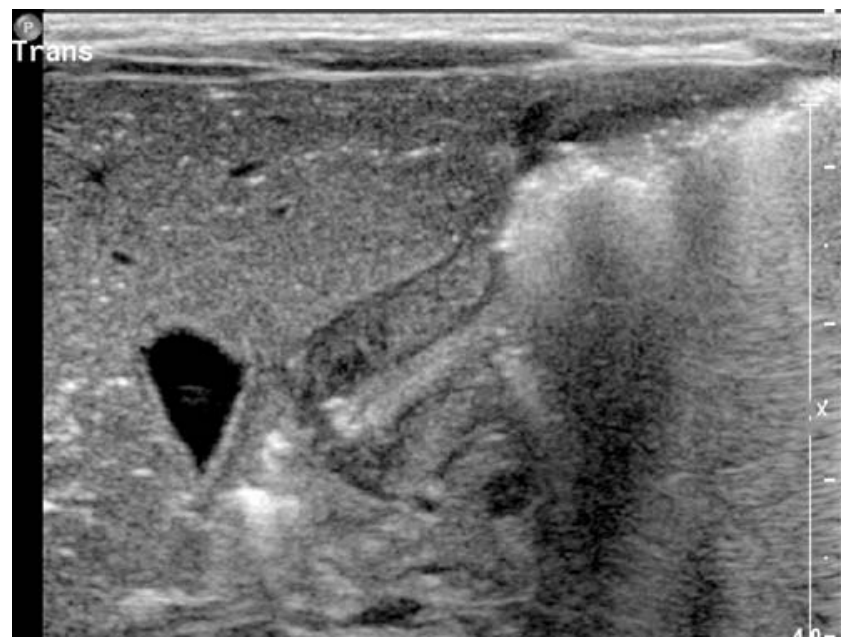

Fig. 1 US image

P. Bhargava $(\bowtie) \cdot$ M. Parisi

Department of Radiology, Children's Hospital \& Regional

Medical Center,

4800 Sand Point Way NE,

Seattle, WA 98105, USA

e-mail: bhargp@u.washington.edu

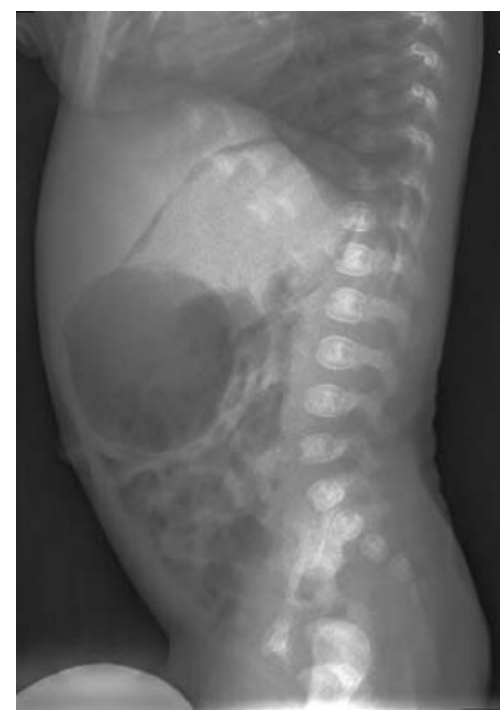

Fig. 2 Lateral abdominal radiograph

duodenal and pyloric stenosis, suggesting a mechanical etiology for the finding. Pyloric stenosis presumably increases intraluminal pressure in the stomach, forcing gas through an intact mucosa [1]. Gastric pneumatosis in the presence of pyloric stenosis is a benign entity with rapid resolution following pyloromyotomy. Awareness of this constellation of findings will prevent unnecessary imaging and postponement of surgery [2].

\section{References}

1. Lester PD, Budge AF, Barnes JC et al (1978) Gastric emphysema in infants with hypertrophic pyloric stenosis. AJR 131:421-423

2. Sarti J, Kennedy A (2006) Portal venous gas in hypertrophic pyloric stenosis. J Pediatr Surg 41:1935-1936 